\section{Molecular Syndromology}

Mol Syndromol 2017;8:325-330

DOI: $10.1159 / 000479455$
Accepted: June 2, 2017

by M. Schmid

Published online: September 7, 2017

\title{
Genetic Counselling Pitfall: Co-Occurrence of an 11.8-Mb Xp22 Duplication and an Xp21.2 Duplication Disrupting IL1RAPL1
}

\author{
Nicolas Chatron ${ }^{\mathrm{a}-\mathrm{c}}$ Lucie Thibault $^{\mathrm{a}}$ James Lespinasse ${ }^{\mathrm{d}}$ Audrey Labalme ${ }^{\mathrm{a}}$ \\ Caroline Schluth-Bolard ${ }^{\mathrm{a}-\mathrm{c}}$ Marianne Till ${ }^{\mathrm{a}}$ Patrick Edery ${ }^{\mathrm{a}-\mathrm{c}}$ Renaud Touraine ${ }^{\mathrm{e}}$ \\ Vincent des Portes ${ }^{c, f, g} \quad G$ Gaetan Lesca ${ }^{a-c}$ Damien Sanlaville ${ }^{a-c}$ \\ ${ }^{a}$ Hospices Civils de Lyon, Service de Génétique, CHU de Lyon, b Equipe GENDEV INSERM U1028, CNRS, UMR5292,

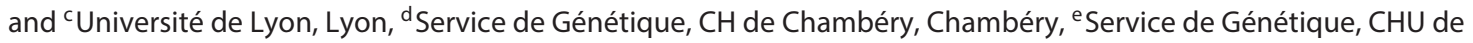 \\ Saint Etienne, Saint Etienne, and ${ }^{\mathrm{f} H}$ ospices Civils de Lyon, Centre de Référence National "Déficiences Intellectuelles \\ de Causes Rares", HFME, and ${ }^{9}$ CNRS UMR 5304, ISC, Bron, France
}

\section{Established Facts}

- Intellectual disability (ID) affects between 1.5 and $2 \%$ of the population with a larger proportion of males due to the number of X-linked genes implicated in ID.

- Mutations in IL1RAPL1 are associated with non-syndromic ID and autism spectrum disorders.

\section{Novel Insights}

- Small CNV detection $(<400 \mathrm{~kb})$ is of medical interest, while next-generation sequencing assays could miss such rearrangements.

- A proportion of patients or families can be affected by 2 different molecular events complicating genetic counselling.

\section{Keywords}

Genetic counselling · IL 1RAPL1 duplication - Intellectual disability $\cdot$ Meiotic recombination $\cdot X$ chromosome

\section{Abstract}

We report a 3-generation family in which 2 Xp copy number variations (CNVs) co-segregate. The proband presented with syndromic intellectual disability. The CNV had been revealed

\section{KARGER}

(C) 2017 S. Karger AG, Basel

E-Mail karger@karger.com

www.karger.com/msy by conventional karyotyping, identifying a large Xp22 duplication causing an $\mathrm{Xp}$ functional disomy. Family studies found that this duplication was inherited from the proband's mother and was also present in one of his sisters. This sister had conventional karyotyping performed during pregnancy with a normal result. Postnatally, her child, the proband's nephew, presented with autism spectrum disorders. aCGH revealed a 339-kb IL1RAPL1 duplication. Overall, the proband, his mother, and one of his sisters all harboured both
Damien Sanlaville

Service de Génétique, Centre de Biologie Est

59 Boulevard Pinel

FR-69677 Bron Cedex (France)

E-Mail damien.sanlaville@chu-lyon.fr 
CNVs, while his other sister and the 2 sons of each sister only carried the ILIRAPL1 intragenic duplication. As seen in this family, we emphasise the importance of small CNV detection, the pathogenicity of ILIRAPLI exonic duplications in male carriers, and the difficulties for genetic counselling with the risk of double diagnosis in a single patient.

(c) 2017 S. Karger AG, Basel

Using whole-genome sequencing, Gilissen et al. [2014] estimated that $62 \%$ of the cases of severe intellectual disability (ID) have a genetic background. ID affects between 1.5 and $2 \%$ of the population in Western countries, especially among males [Leonard and Wen, 2002]. The high prevalence among males could be due to the location of the genes involved in ID in the X chromosome. Variants in genes located in the $\mathrm{X}$ chromosome are expected to account for about $10 \%$ of all male cases of ID [Ropers, 2008, 2010; Gécz et al., 2009]. More than 100 genes have already been identified in X-linked ID. Among these, IL1RAPL1, located in the $\mathrm{Xp} 21.2$ chromosomal region, is mainly expressed in cerebral structures involving memory and encodes a 696-amino acid transmembrane protein involved in spinogenesis [Hayashi et al., 2013]. It is now well recognised that mutations in ILIRAPL1 are associated with non-syndromic ID and autism spectrum disorders [Piton et al., 2008].

In this report, we present a family with several males with different degrees of ID. Cytogenetic investigation identified 2 duplications located in the same X chromosome. A large Xp22.31p22.12 duplication was first identified by standard karyotype analysis. Years later, a small intragenic IL1RAPL1 duplication was found using array $\mathrm{CGH}(\mathrm{aCGH})$, once again demonstrating the superiority of aCGH compared to karyotyping in the diagnosis of developmental disorders [Hochstenbach et al., 2009; Miller et al., 2010]. In this family, segregation of these Xp copy number variations (CNVs) was modified over 2 generations explaining different phenotypes in males and leading to errors in genetic counselling.

\section{Case Reports}

The pedigree of the family is shown in Figure 1.

\section{Patient II.5}

Patient II. 5 was the first to be addressed for genetic testing. This 44-year-old patient had profound ID. His head circumference at birth was $33 \mathrm{~cm}$ (10th percentile). He had delayed developmental milestones with hypotonia and strabismus. He walked at 2.5 years of age and had no verbal skills with very poor interaction skills. He walked with a slightly broad-based gait and had stereotyped movements. He had a very quiet personality. Dysmorphic features included pectus excavatum, hypertelorism, epicanthus, a flat nasal bridge, arched eyebrows, retrognathia, fleshy lips, and large ears (Fig. 2).

\section{Patient III.1}

This 11-year-old boy was born at term after an uneventful pregnancy. At birth, his weight was 3,415 g (40th percentile), height was $52 \mathrm{~cm}$ (75th percentile), and head circumference was $36 \mathrm{~cm}$ (50th percentile). Apgar score was 10/10. Early psychomotor development was slightly delayed. He walked at 17 months of age. He had moderate fine motor impairment and early social interaction problems contrasting with normal language acquisition. He needed sameness of environment and had perseverative behaviour, with a low frustration tolerance. He was diagnosed with visuospatial dyspraxia and attention deficit at 6.5 years of age as well as Asperger syndrome (using the ADOS and ADI-R scales) at 7 years of age. On the WISC-IV, at 10 years of age, on methylphenidate 15 mg per day, his verbal comprehension index was 86, perceptual reasoning index was 52 , working memory index was 60 , and processing speed index was 59 . On clinical examination, he had no neurological or dysmorphic features. EEG and brain MRI, performed respectively at 6 and 8 years of age, were normal. Fragile X genetic analysis and metabolic screening (T3-T4-TSH, urinary organic acids, orotic acid, creatinine, and guanidinoacetate, blood aminoacids) were normal.

\section{Patient III.5}

This 6.5-year-old child was born after an uneventful pregnancy. At birth, weight was 3,320 g (35th percentile), height was $50 \mathrm{~cm}$ (50th percentile), and head circumference was $36.5 \mathrm{~cm}$ (64th percentile). Early psychomotor development was normal. He walked at 15 months, and language started at 18 months. He had mild problems with social interaction, but did not meet criteria for pervasive developmental disorder on autism-standardised scales. At school age, he was diagnosed with dyspraxia and attention deficit with hyperactivity, which improved on methylphenidate $20 \mathrm{mg}$ per day. On the WISC-IV, at 6.5 years of age, his verbal comprehension index was 96, perceptual reasoning index was 67, working memory index was 60 , and processing speed index was 50 . On clinical examination, he had no neurological or dysmorphic features, except pes planus.

\section{Methods}

\section{Karyotyping}

Standard GTG- and RHG-banded karyotypes obtained from peripheral blood were determined for patient II.5 using standard procedures.

\section{Array $\mathrm{CGH}$}

QIAamp DNA Blood Midikit (Qiagen, Courtaboeuf, France) was used for DNA extraction. Oligonucleotide aCGH was performed with an 180,000-oligonucleotide microarray (SurePrint G3 Human CGH Microarray Kit, 4×180K, AMADID: 022060, Agilent Technologies, Santa Clara, CA, USA). The median probe spacing
Chatron et al. 


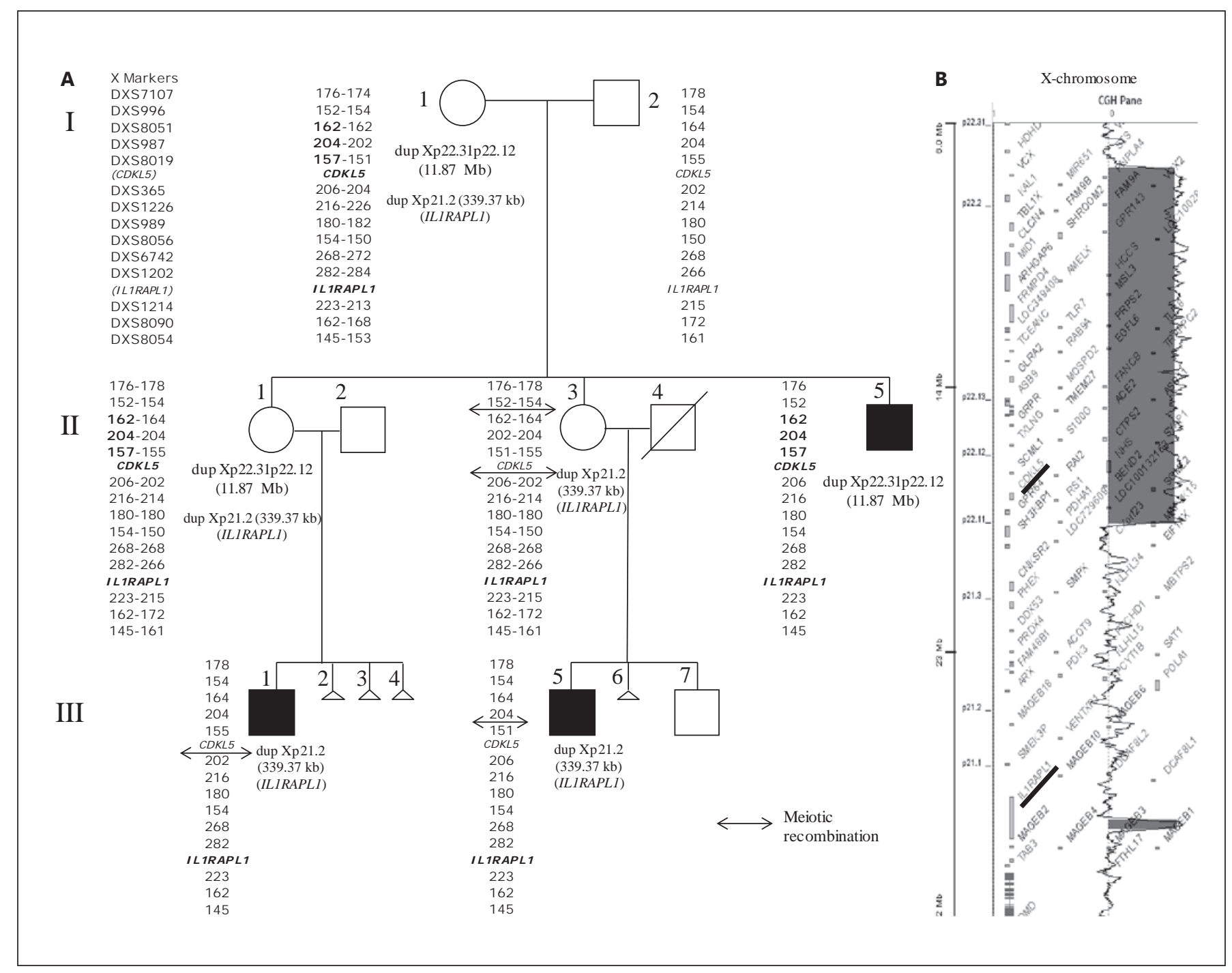

Fig. 1. Family pedigree displaying CNV-carrier status. A All live-born patients were tested either by aCGH or by qPCR. Haplotype analysis is shown. Duplications are represented in bold and recombinations by arrows. B aCGH profile of the short arm of the X chromosome for patient II.1.

of this microarray is about $13 \mathrm{~kb}$. The aCGH procedures were performed in accordance with the manufacturer's instructions. The $180 \mathrm{~K}$ slides were scanned on an Agilent DNA Microarray Scanner and images were extracted with Feature Extraction software (10.7.3.1). Data analysis was carried out with Genomic Workbench 5.0.14 software (Agilent Technologies). The following parameters were used for interpretation: ADM-2, threshold: 5.0, window: $0.2 \mathrm{Mb}$, cutoff: 0.25 . A CNV was validated if an abnormal $\log _{2}$ ratio was obtained for at least 3 contiguous probes. The aCGH results were analysed using the UCSC hg19 assembly (https://genome-euro.ucsc.edu/).

\section{Quantitative PCR}

Quantitative PCR (qPCR) was performed on genomic DNA, with 2 primer pairs amplifying unique sequences within IL1RAPL1 and within $A D O R A 2 B$ as the reference gene and the QuantiTect SYBR Green PCR Kit (Qiagen). Experiments were conducted on a Light Cycler 2000 (Roche Applied Science, Indianapolis, IN, USA).

\section{Haplotype Analysis}

Microsatellite markers spread throughout the X chromosome and particularly covering the 2 regions of interest were studied (from Xp telomere to centromere): DXS7107, DXS996, DXS8051, DXS987, DXS8019, DXS365, DXS1226, DXS989, DXS8056, DXS6742, DXS1202, DXS1214, DXS8090, and DXS8054. X-chromosome inactivation was studied by the methyl-PCR method at the FMR1 locus using an in-house protocol and after treatment of DNA by the EpiTect Bisulfite kit (Qiagen). Fragment analyses were run on a sequencing machine CEQ-2000XL (Beckman Coulter, Brea, CA, USA). 
Fig. 2. Photographs of patient II.5 at 3 months $(\mathbf{A}), 5$ years $(\mathbf{B}), 8$ years $(\mathbf{C})$, and 35 years of age (D).
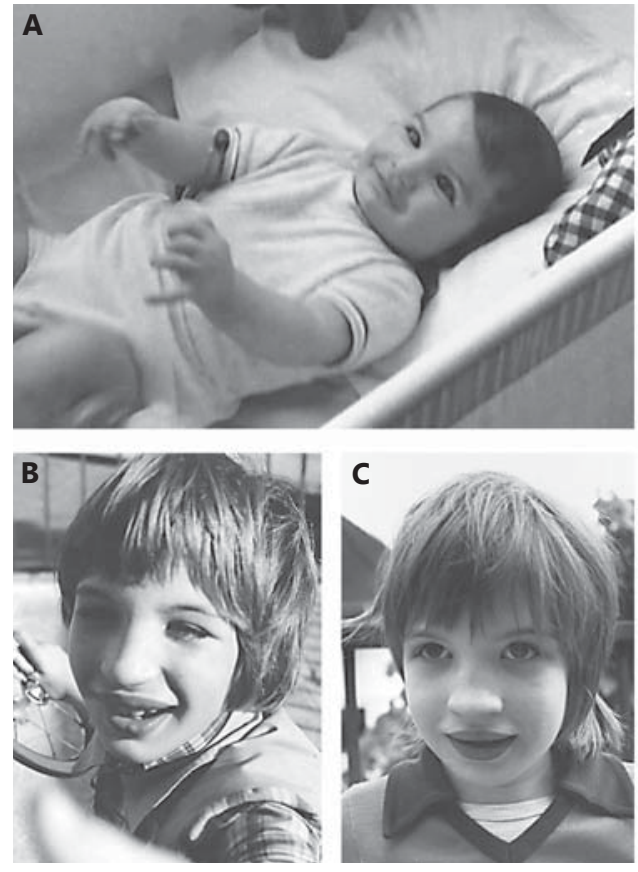

\section{Results}

Chronologically, several years ago, standard karyotype analysis for patient II.5 showed an Xp22.31p22.12 duplication, 46, Y, dup (X)(p22.31p22.12); aCGH was not an established method at this time. His mother (I.1) and one of his sisters (II.1) were asymptomatic carriers, whereas the other sister (II.3) had a normal karyotype. Consequently, a prenatal exploration by chorionic villus sampling was proposed and performed during pregnancy of patient II.1. The Xp22.31p22.12 duplication was not found by standard karyotyping, leading to a reassuring genetic counselling. However, postnatally, aCGH performed in patient III. 1 because of psychomotor delay and behaviour troubles, found a 339-kb duplication in Xp21.2. This intragenic duplication encompasses exons 4 and 5 of IL1RAPL1, $\operatorname{arr}[$ GRCh38] Xp21.2(29318390_29657768)×2 . qPCR and/or aCGH analysis showed that his mother (II.1), his aunt (II.3), his cousin (III.5), and his grandmother (I.1) were all carriers of this duplication, just as the proband (II.5). All other male patients of the family, who had no neurodevelopment impairment, were not carriers of this Xp21.2 duplication. aCGH also delineated the Xp22.31p22.12 duplication to $11.8 \mathrm{Mb}$ between the positions 7666592 and 19446130 (GRCh38). This large duplication spans 59 genes, including 15 pathogenic OMIM genes (http://www.omim.org/).
Patients I.1 and II.1, both harbouring the large Xp22.31p22.12 duplication, had complete skewed Xchromosome inactivation (0:100\%), whereas patient II.3 (carrying only the IL1RAPL1 duplication) had a normal $\mathrm{X}$-chromosome inactivation pattern.

Microsatellite analysis found that in I.1, II.1, and II.5, the $2 \mathrm{CNV}$ s were located in the same X chromosome and that a double crossing-over had occurred during grandmaternal meiosis in patient II.3, both upstream and downstream of the large Xp22.31p22.12 duplication. During meiosis in patient II.1, a simple recombination occurred between the markers DXS365 and DXS1226, leading to a chromosome without a large Xp22 duplication in patient III.1, but in which the IL1RAPL1 duplication was maintained (data not shown).

\section{Discussion}

The clinical features seen in patient II. 5 could be explained by the $11.8-\mathrm{Mb}$ Xp22.31p22.12 duplication leading to a functional Xp22 disomy. Indeed, the patient presents with the key phenotypic features related to Xp functional disomy as described by Hunter et al. [2009]: cognitive impairment, hypotonia, hypertelorism, and ear abnormalities. Considering the 59 genes duplicated, it is difficult to obtain a precise genotype/phenotype 
correlation for each phenotypic trait as it has already been the case in similar findings of large Xp functional disomies [Lintas et al., 2016]. However, among others, we could note the presence of a single CDKL5 gene, while CDKL5 duplications have already been reported as being responsible for autistic behaviour and development and speech delay in both males and females in case of random X-chromosme inactivation [Szafranski et al., 2015]. We observed complete skewed X-chromosome inactivation in both female carriers of this duplication (patients I.1 and II.1).

The 2 male patients of this family presenting with the same isolated intragenic IL1RAPL1 duplication (III.1and III.5) share a similar phenotype, including autism spectrum disorders, short attention span, and dyspraxia without ID as well as dysmorphic features. Froyen et al. [2007] and Zhang et al. [2009] both reported a case of IL1RAPL1 duplication associated with ID, but in both cases, the CNV encompassed other XLID genes such as CASK and DMD. Four patients in the DECIPHER database $(274669,277292,249698$ and 280583; https://decipher.sanger.ac.uk/) harbour an overlapping intragenic IL1RAPL1 duplication. They all present with developmental delay as patients from the database harbouring an overlapping deletion.

Interestingly, 2 apparently unrelated Japanese families carrying $2 \mathrm{CNVs}$ close to those found in the family presented herein, namely a $737-\mathrm{kb}$ duplication in Xp22.2 (chrX:16880008-17617255 [GRCh38]) and a 100-kb duplication in IL1RAPL1 in Xp21.3 (chrX:2869347728793925 [GRCh38]), have been reported by Honda et al. [2010] These patients harboured variable clinical phenotypes: autistic features and speech delay with moderate ID for the first family and West syndrome with absent speech and severe ID for the second one. Using hg38 UCSC Genome Browser (http://grch37.ensembl.org/index.html), no repeated genomic elements potentially promoting rearrangements at breakpoint loci were found, and there is no argument for a recurrent mechanism for the 2 duplications. Finally, Utine et al. [2014] reported a small IL1RAPL1 duplication also encompassing exons 4 and 5 in a patient with syndromic developmental delay and microcephaly, corpus callosum agenesis, short stature, and facial dysmorphism. He was born to consanguineous parents, raising the risk for an additional unknown diagnosis which could explain the phenotypic differences compared to other patients carrying IL1RAPL1 variants [Utine et al., 2014].

The X-linked Mental Retardation 21 OMIM phenotype linked to IL1RAPL1 alterations (OMIM 300143) is defined by variable ID and possible additional features such as facial dysmorphism and autistic traits. RamosBrossier et al. [2015] have demonstrated that a loss-offunction mechanism impairing synaptogenesis was consecutive to 2 different single nucleotide variations and an exonic deletion. We hypothesise that this intragenic IL1RAPL1 duplication leads to haploinsufficiency, either by a truncation or by a conformational change in the protein, as described for other genes [Liedén et al., 2014; Schwaibold et al., 2014]. It is of note that in silico reconstruction of tandem duplication of exons 4 and 5 (NM_014271.3(IL1RAPL1_v001):c.363_703dup) leads to a premature stop codon.

In 2012, Jobanputra et al. addressed the question of the influence of CNVs in the X-chromosome inactivation process. The authors suggest that even if skewed Xchromosome inactivation can rarely be related to a $\mathrm{CNV}$, the size of the CNV may influence the X-inactivation ratio. Larger CNVs seemed to be more frequently associated with complete skewed $\mathrm{X}$ inactivation than small CNVs, whatever the precise gene content is. This is consistent with the family reported herein, in which the 2 females bearing the large $\mathrm{CNV}$ in the $\mathrm{X}$ chromosome (I.1 and II.1) had a complete skewed X-chromosome inactivation, whereas patient II.3, bearing only the small intragenic IL1RAPL1 CNV, had random X inactivation. This has also been recently reported in another family with intragenic IL1RAPL1 duplication [Laino et al., 2016].

One important aspect for genetic counselling based on this familial observation is the risk of a double molecular event as it has been described by exome sequencing; $1.4 \%$ of the patients have more than a single molecular diagnosis [Yang et al., 2014]. Indeed, the large Xp22 duplication explained the phenotype of patient II.5 and was logically targeted for familial inquiry and prenatal testing thereafter. However, the result obtained by conventional karyotype analysis was insufficient for appropriate genetic counselling. An aCGH would have revealed the risk of occurrence of the 2 $\mathrm{CNV}$ s in the offspring, suggesting adequate prenatal genetic testing.

In practice, the occurrence of a second pathogenic $\mathrm{CNV}$ or even another pathogenic variation and possible meiotic recombinations in a family must be kept in mind for genetic investigations and counselling. 


\section{Statement of Ethics}

Experiments were conducted in accordance with the Declaration of Helsinki. All procedures were carried out with the adequate understanding, and written informed consent was obtained from the patients or their parents.

\section{Disclosure Statement}

The authors declare no conflicts of interest.

\section{References}

Froyen G, Van Esch H, Bauters M, Hollanders K, Frints SG, et al: Detection of genomic copy number changes in patients with idiopathic mental retardation by high-resolution X-array-CGH: important role for increased gene dosage of XLMR genes. Hum Mutat 28:10341042 (2007)

Gécz J, Shoubridge C, Corbett M: The genetic landscape of intellectual disability arising from chromosome X. Trends Genet 25:308316 (2009).

Gilissen C, Hehir-Kwa JY, Thung DT, van de Vorst $\mathrm{M}$, van Bon BW, et al: Genome sequencing identifies major causes of severe intellectual disability. Nature 511:344-347 (2014).

Hayashi T, Yoshida T, Ra M, Taguchi R, Mishina M: IL1RAPL1 associated with mental retardation and autism regulates the formation and stabilization of glutamatergic synapses of cortical neurons through RhoA signaling pathway. PLoS One 8:e66254 (2013).

Hochstenbach R, van Binsbergen E, Engelen J, Nieuwint A, Polstra A, et al: Array analysis and karyotyping: workflow consequences based on a retrospective study of $36,325 \mathrm{pa}-$ tients with idiopathic developmental delay in the Netherlands. Eur J Med Genet 52:161-169 (2009).

Honda S, Hayashi S, Imoto I, Toyama J, Okazawa $\mathrm{H}$, et al: Copy-number variations on the $\mathrm{X}$ chromosome in Japanese patients with mental retardation detected by array-based comparative genomic hybridization analysis. J Hum Genet 55:590-599 (2010).
Hunter M, Bruno D, Amor DJ: Functional disomy of proximal Xp causes a distinct phenotype comprising early hypotonia, hypertelorism, small hands and feet, ear abnormalities, myopia and cognitive impairment. Am J Med Genet A 149A:1763-1767 (2009).

Jobanputra V, Levy B, Kinney A, Brown S, Shirazi $\mathrm{M}$, et al: Copy number changes on the $\mathrm{X}$ chromosome in women with and without highly skewed X-chromosome inactivation. Cytogenet Genome Res 136:264-269 (2012).

Laino L, Bottillo I, Piedimonte C, Bernardini L, Torres B, et al: Clinical and molecular characterization of a boy with intellectual disability, facial dysmorphism, minor digital anomalies and a complex IL1RAPL1 intragenic rearrangement. Eur J Paediatr Neurol 20:971-976 (2016).

Leonard H, Wen X: The epidemiology of mental retardation: challenges and opportunities in the New Millennium. Ment Retard Dev Disabil Res Rev 8:117-134 (2002).

Liedén A, Kvarnung M, Nilssson D, Sahlin E, Lundberg ES: Intragenic duplication - a novel causative mechanism for SATB2-associated syndrome. Am J Med Genet A 2014 164A:3083-3087 (2014)

Lintas C, Picinelli C, Piras IS, Sacco R, Gabriele S, et al: Xp22.33p22.12 duplication in a patient with intellectual disability and dysmorphic facial features. Mol Syndromol 6:236-241 (2016).

Miller DT, Adam MP, Aradhya S, Biesecker LG, Brothman AR, et al: Consensus statement: chromosomal microarray is a first-tier clinical diagnostic test for individuals with developmental disabilities or congenital anomalies. Am J Hum Genet 86:749-764 (2010).
Piton A, Michaud JL, Peng H, Aradhya S, Gauthier J, et al: Mutations in the calcium-related gene IL1RAPL1 are associated with autism. Hum Mol Genet 17:3965-3974 (2008).

Ramos-Brossier M, Montani C, Lebrun N, Gritti L, Martin C, et al: Novel IL1RAPL1 mutations associated with intellectual disability impair synaptogenesis. Hum Mol Genet 24:11061118 (2015).

Ropers HH: Genetics of intellectual disability. Curr Opin Genet Dev 18:241-250 (2008).

Ropers HH: 2010. Genetics of early onset cognitive impairment. Ann Rev Genomics Hum Genet 11:161-187 (2010).

Schwaibold EM, Smogavec M, Hobbiebrunken E, Winter L, Zoll B, et al: Intragenic duplication of EHMT1 gene results in Kleefstra Syndrome. Mol Cytogenet 7:74 (2014).

Szafranski P, Golla S, Jin W, Fang P, Hixson P, et al: Neurodevelopmental and neurobehavioral characteristics in males and females with CDKL5 duplications. Eur J Hum Genet 23: 915-921 (2015).

Utine GE, Haliloğlu G, Volkan-Salancı B, Cetinkaya A, Kiper PÖ, et al: Etiological yield of SNP microarrays in idiopathic intellectual disability. Eur J Paediatr Neurol 18:327-337 (2014).

Yang Y, Muzny DM, Xia F, Niu Z, Person R, et al: Molecular findings among patients referred for clinical whole-exome sequencing. JAMA 312:1870-1879 (2014)

Zhang Z, Yagi M, Okizuka Y, Awano H, Takeshima Y, Matsuo M: Insertion of the IL1RAPL1 gene into the duplication junction of the dystrophin gene. J Hum Genet 54:466473 (2009) 